\title{
INTRODUCCIÓN DE BOMBUS (BOMBUS) TERRESTRIS (LINNAEUS, 1758)) (HYMENOPTERA: APIDAE) EN LA REGIÓN DE MAGALLANES: POTENCIAL RIESGO PARA LAS ABEJAS NATIVAS
}

\author{
BOMBUS (BOMBUS) TERRESTRIS (LINNAEUS, 1758)) (HYMENOPTERA: APIDAE) \\ INTRODUCTION IN THE MAGELLAN REGION: POTENTIAL RISK FOR THE NATIVE BEES ${ }^{1}$
}

Vicente Pérez ${ }^{2}$

Hymenoptera es el megaorden más importante desde el punto de vista de la contribución de sus especies en la polinización de las plantas. Entre los agentes más importantes están la abeja de miel (Apis mellifera Linnaeus, 1758), en primer lugar, y luego el grupo de los abejorros (Bombus). Éstos están entre los más destacados polinizadores de las regiones temperadas, por una parte, debido a la forma, tamaño y estructura de su cuerpo y por otra, a los tamaños de sus probóscides, la capacidad para "sonicar" ("polinización vibrátil", empleo de sonidos para liberar el polen de anteras poricidas o dehiscentes como las de las Solanáceas), la pilosidad densa que les permite transferir mayor número de granos de polen por visita, la actividad durante largos períodos y la adaptabilidad a una amplia variedad de temperaturas y tipos de climas debido a que son capaces de generar calor metabólico (Buchmann, 1983, Willmer et al., 1994, NAPPC, 2006, Morales, 2007).
La introducción de abejorros para la polinización en invernaderos es un hecho generalizado en los años recientes y su demanda se incrementa anualmente. Entre ellos tenemos al abejorro europeo Bombus terrestris (Linnaeus, 1758), que se está utilizando en diversas partes del mundo por su gran eficacia como polinizador (Yoon et al., 2009). Es uno de los abejorros más comunes de Europa, y, entre las 250 especies de abejorros descritas, $B$. terrestris es una de las especies que ha sido más estudiada (Coppée, 2010). Por sus hábitos alimentarios es altamente generalista. Así, para Francia y Bélgica, Rasmont (1988) reportó más de trescientas especies de plantas visitadas por él (fide Coppée, $2010^{3}$ ).

El interés por utilizar esta especie como polinizadora en invernaderos no declina (Velthuis $\&$ Doorn, 2006). Actualmente es una de las cinco especies de Bombus que se crían comercialmente y la más extensamente utilizada para polinizar más de 25 cultivos, tanto en invernaderos (tomate, frutilla,

\footnotetext{
${ }^{1}$ Corresponde al Programa de Investigación Biodiversidad de los insectos en la región de Magallanes.

${ }^{2}$ Laboratorio de Entomología, Instituto de la Patagonia, Universidad de Magallanes, Punta Arenas, Chile.

${ }^{3}$ Coppée, A. 2010. Bombus terrestris. A complex of species or a rare complex? Intraspecific pheromonal and genetics variations

on Bombus terrestris (L.). Impacts on the speciation. Universite de Mons. Thesis submitted in fulfillment of the degree of

Doctor in Sciences. Supervisor: Prof. P. Rasmont. http//www.atlashymenoptera.net/biblio/Coppee_Ph_D_complet.pdf
} 
pimiento) como a campo abierto (kiwi, almendro, manzano y palto) (Morales, 2007).

Inicialmente, Bombus terrestris fue importado por diversos países con el propósito de emplearlo como polinizador de tomates en invernaderos, para lo cual hay una amplia oferta de criadores del abejorro, que los ofrecen comercialmente como "colonias" que se despachan en cajas que se transportan a los más diversos lugares (Matsumura et al., 2004). En el Reino Unido se importan 10.000 "colonias" cada año (Goulson et al., 2008). Suelen escapar de los invernaderos y formar sociedades silvestres. Así, en Japón se han naturalizado (Matsumura et al.,2004), aun cuando se emitieron advertencias sobre el peligro de su propagación (Dafni, 1998) y se comprobó posteriormente el efecto negativo que produjo en los abejorros nativos (Inoue et al., 2008).

La oportunidad de disminuir los costos de producción de frutos, mejorando la cantidad y calidad, con la importación de abejorros, ha estimulado a una industria que mueve miles de millones de dólares al año. Casi todas las especies de Bombus que se utilizan como polinizadores pertenecen al subgénero Bombus sensu stricto, los cuales incluyen a la mayoría de las especies polinizadoras comerciales de abejorros (Willams et al., 2012).

El objetivo de esta contribución es presentar todos los antecedentes sobre esta especie de abeja, muy eficiente polinizador en el viejo continente, que, introducido en Chile, mantiene sus cualidades pero está produciendo efectos negativos en las abejas nativas y que, recientemente, también ha llegado a la región de Magallanes. Están en riesgo el único abejorro (Bombus dahlbomii Guérin-Meneville, 1835) y las 14 especies de abejas solitarias que habitan nuestra zona.

Bombus (Bombus) terrestris (L.) en Chile

Esta abejorro fue introducido en Chile en 1997 (Montalva et al. 2008) como agente polinizador de tomates en invernaderos por ser un eficiente polinizador; y en 2001 se demostró que no era un eficiente polinizador del palto. Los primeros ejemplares recolectados en campo abierto fueron los de Quillota en 1998; y en el sector cordillerano de Santiago se han observado ejemplares volando a los $3.400 \mathrm{~m}$ s.n.m. (Montalva 2012). Esta adaptación a las alturas coincide con la apreciación de Briscoe \& Chittka
(2001) en cuanto a que las especies de un solo género (Bombus) se encuentran desde Groenlandia hasta la cuenca del Amazonas, y desde el nivel del mar hasta altitudes aproximadas a los $5800 \mathrm{~m}$ s.n.m. en los Himalayas. Observaciones de campo indicarían que los principales polinizadores de las plantas de alta montaña son himenópteros del género Bombus (Bosch et al., 1997). En la actualidad, esta especie se ha naturalizado y está distribuida ampliamente en nuestro país (Montalva et al., 2011). En Chile, su distribución se extendía desde la región de Coquimbo a la región del General Carlos Ibáñez del Campo, encontrándose también en la isla Grande de Chiloé (Montalva, 2012). Desde Chile, Bombus terrestris invadió Argentina (Morales, 2007), siendo registrada su presencia por Torreta et al. (2006).

Bombus terrestris posee el cuerpo negro con bandas pilosas amarillas, diferenciándose de otras especies por el color blanquecino de la pilosidad del extremo del abdomen; es una abeja grande, cuyas reinas miden 2-2,7 cm de longitud y las obreras 1-1,5 $\mathrm{cm}$ (Project Noah, 2013); tiene un mesosoma con una banda transversal amarilla y el metasoma con dos bandas transversales: una amarilla contigua al pedicelo y una blanca en el extremo distal (Montalva 2012); la probóscide de la reina es de unos $10 \mathrm{~mm}$ de largo, siendo más corta la de las obreras (Project Noah, 2013). Sus sociedades pueden llegar a 200 individuos (Montalva, 2012).

De manera similar a las otras especies de Bombus, sólo las reinas sobreviven el invierno. Emergen en la primavera, hacen acopio de energía con el néctar que liban y buscan un lugar para anidar (una cavidad o un nido abandonado de roedor, murallas, bodegas, huecos o espacios entre matorrales) (Montalva, 2012). Puede desplazarse y volver a su nido desde una distancia aproximada de 13 kilómetros, aunque lo frecuente es que forrajeen dentro de un radio de $5 \mathrm{~km}$ de su nido (Project Noah, 2013).

Ruz \& Herrera (2001) encontraron que el tiempo de forrajeo por flores, en vegetación nativa y no nativa, era menor en Bombus terrestris que en Bombus dahlbomii Guérin, el más grande de los abejorros nativos de Chile. El traslapo de la distribución de estas dos especies podría indicar que exista competencia entre ambas especies de abejorros (Montalva et al., 2011).

Por otro lado, cuando se introdujo por primera 
vez a Bombus terrestris en nuestro país, parecía que las regulaciones sanitarias de importación controlaban cuidadosamente los peligros potenciales de este proceso, como la introducción de algún organismo patógeno acompañante que pudiera afectar a las especies nativas de abejas y, especialmente a las de alta importancia económica tales como la abeja de miel (Apis mellifera Linnaeus, 1758) (Estay, 2007, Montalva et al., 2008). Sin embargo, ha quedado demostrado que tales medidas no fueron efectivas. Por ejemplo, el ácaro europeo Kuzinia laevis (Dujardin, 1849) (Acarina: Acaridae) fue detectado en Chile, a partir de especímenes asociados a una reina hibernante de Bombus terrestris en la ciudad de Santiago, Región Metropolitana (Allendes \& Montalva, 2011). Aunque es probable que esta especie comensal no constituya un riesgo para las abejas nativas, existe la posibilidad que otras especies de ácaros más peligrosos, asociados con $B$. terrestris puedan sortear el control sanitario (Goka et al., 2001, Montalva et al. 2008); los protistas parásitos Crithidia bombi (Kinetoplastida) y Apicystis bombi (Apicomplexa) fueron detectados en individuos de $B$. terrestris en el noreste de la región Patagónica argentina, que habían penetrado desde Chile, 3 $\mathrm{km}$ al oeste de San Carlos de Bariloche (Plischuk \& Lange, 2009).

Con la introducción y dispersión de Bombus exóticos se ha producido la transmisión de organismos patógenos, como diversos parásitos, bacterias y otros, que han atacado y diezmado las poblaciones de los abejorros nativos (Schmidt-Hempel 1998).

Estos antecedentes preliminares sugieren que las poblaciones de Bombus terrestris, introducidas artificialmente, habrían tenido un impacto negativo en las especies polinizadoras autóctonas o de larga data establecidas. Muchas publicaciones reflejan claramente, incluso en sus títulos, esta preocupación desde el punto de vista de la conservación y también de la economía local afectada (van der Steen 2000, O'Toole 1994, Dafni et al. 1996, Goka 1998, Thorp 1996, Ono 19974, Hingston \& McQuillan 1999, Ruz 2002, Goulson 2003, Matsumura et al. 2004, Yourth \& Schmidt-Hempel 20045, NAPPC 2006, Morales 2007). Castro (2012) ha expresado muy bien "la difícil situación del abejorro más austral del mundo", el Bombus dahlbomii, habitante de Chile y Argentina, que ha sido el punto central de reuniones científicas (Arbetman et al., 2010 6 , 2012 ${ }^{7}$; Morales et al. $\left.2010^{8}, 2011\right)^{9}$ y conferencias (Arbetman et al. $2012^{7}$, Morales et al. 2012 $)^{10}$.

En general, existen a lo menos cinco efectos negativos que generaría la introducción de abejas no nativas: competencia con los visitantes florales nativos, competencia con las especies nativas por los sitios de nidificación, transmisión a los insectos autóctonos de organismos patógenos, cambios en la producción de semillas de las plantas nativas y polinización de malezas introducidas (Goulson, 2003, fide Morales 2007).

Bombus (Bombus) terrestris en la región de Magallanes

Vía introducción voluntaria por el Instituto Nacional de Investigaciones Agrícola (INIA), esta abeja ya se encuentra en la Región de Magallanes, que la mantiene experimentalmente como polinizador en

\footnotetext{
${ }^{4}$ Ono, M. 1997. Ecological implications of introduced Bombus terrestris, and significance of domestication of Japanese native bumblebees (Bombus spp.). In Proceedings, International Workshop Biological Invasions of Ecosystem by Pests and Beneficial Organisms, pp.244-252. National Institute of Agro-Environmental Sciences, Ministry of Agriculture, Forestry, Fisheries. Tsukuba, Japan.

${ }^{5}$ Yourth, C.P. \& P. Schmidt-Hempel . 2004. Virulence and transmission of Crithidia bombi in bumble bees. Proceedings of the First Conference of Apidology, pp. 83-84. Udine.

${ }^{6}$ Arbetman, M., I. Neeus, C. Morales, G. Smagghe \& M. Aizen. 2010. La transmisión de parásitos patógenos entre abejorros introducidos y el nativo: un mecanismo complementario a la competencia que explicaría el colapso poblacional de Bombus dahlbomii en el noroeste de la Patagonia. 2do. Taller Iberoamericano sobre degradación de hábitats y funcionamiento de interacciones planta-animal. Bariloche, Argentina.

7 Arbetman, M., C.L. Morales, G. Smagghe, M.A. Aizen \& I. Meeus. 2012. Detección e identificación molecular de parásitos patógenos en abejorros de la Patagonia de Argentina. VIII Congreso Argentino de Entomología. Bariloche, Argentina.

${ }^{8}$ Morales, C.L. \& M.A. Aizen. 2010. Reemplazo ecológico del abejorro nativo (Bombus dahlbomii) por abejorros introducidos (B. terrestris y B. ruderatus). 2do. Taller Iberoamericano sobre degradación de hábitats y funcionamiento de interacciones planta-animal. Bariloche, Argentina

${ }^{9}$ Morales, C.L., M. Arbetman \& M.A.Aizen 2011. La globalización en el mundo de los polinizadores: impacto de abejorros en la biota nativa. Simposio Impacto de especies invasoras en la Patagonia. II Jornadas de Ciencias Naturales de la Patagonia. Esquel, Argentina.

${ }^{10}$ Morales, C.L., M. Arbetman \& M.A. Aizen. 2012. Una exploración sobre las posibles causas locales y regionales de la declinación global de especies de abejorros (Bombus). VIII Congreso Argentino de Entomología. Bariloche, Argentina.
} 
invernaderos en la provincia de Magallanes. También se encuentra en la provincia de Última Esperanza, traída por agricultores privados.

Hasta el momento, no se tienen noticias de la fuga de reinas de Bombus terrestris desde los invernaderos hacia el campo abierto y de su posible asilvestración.

El clima riguroso en Magallanes, con la constante del viento, temperatura y pluviosidad variables, mantiene una apidofauna de escasa biodiversidad. Así, en referencia a las abejas, han fracasado los intentos de naturalizar a la abeja de miel (Apis mellifera L.) (Pérez \& Petersen, 1989), y de las 424 especies de abejas registradas para el territorio nacional, no habría en Magallanes más de una quincena (Montalva \& Ruz, 2010). Bombus (Fervidobombus) dahlbomii Guérin-Meneville, 1835, es la única especie social anual que habita estas altas latitudes. Las otras son de comportamiento solitario.

\section{Consideraciones finales}

Aun cuando las consecuencias de la introducción de Bombus terrestris a Magallanes aún son especulativas, están en riesgo el único abejorro nativo de Chile (Bombus dahlbomii GuérinMeneville, 1835) y las 14 especies de abejas solitarias que habitan en esta región. Sin embargo, los antecedentes recopilados son claros en mostrar los riesgos de dicha introducción. El desplazamiento de las abejas nativas por competencia por los recursos y la transmisión de patógenos serían los principales peligros para la apifauna regional. Además, son particularmente propensas las especies solitarias de la región, debido a los bajos tamaños poblacionales que éstas presentan.

Como destaca Montalva (2001), junto con las ventajas de la globalización se presentan también desventajas a las cuales "muchas veces no se les da la importancia que merecen, especialmente por la falta de comprensión de la sociedad". En este caso estamos ante los efectos que puede tener la introducción de un abejorro foráneo sobre la apidofauna regional. En este sentido, la introducción de Bombus terrestris a la región de Magallanes no tiene justificación alguna si se considera que Magallanes es posiblemente la menos agrícola de las regiones del sur de Chile, y se abastece de productos vegetales para el consumo de la población parcialmente por los cultivos a campo abierto y minoritariamente por el de invernaderos, dependiendo mayoritariamente de los envíos del resto del país y, ocasionalmente, de los proveniente de la República Argentina.

El desarrollo de la conciencia pública sobre el problema de la disminución y la posible extinción de especies biológicas se atribuye mayoritariamente al concepto del Red Data Book (RDB) por Sir Peter Scott durante 1960, cuyo foco estuvo enfocado en los primeros RDBs hacia los vertebrados, invertebrados $y$ plantas y que fue adoptado en diversos niveles por varios países. Sin embargo, a pesar de que en 1990 la Lista Roja comprendía 4.452 especies de animales, de las cuales 1.083 correspondían a insectos (Groombridge, 1992) y el tema de la biodiversidad aparece como dominante y recurrente, no se refleja en publicaciones recientes, y los insectos como taxón ni siquiera son mencionados (Squeo et al., 2010).

Lamentablemente, los insectos no son considerados en las Declaraciones de Impacto Ambiental ni en los Estudios de Impacto Ambiental del Sistema de Evaluación de Impacto Ambiental exigidos antes de un emprendimiento que pueda afectar el ambiente, posiblemente por los escasos antecedentes que se tienen sobre el rol que juegan en la economía de la naturaleza, pero que pueden afectarnos en el lejano plazo, cuando el ambiente se haya fragmentado y las transformaciones antrópicas afecten de manera irreversible la biodiversidad ligada a estos animales, el mayor grupo natural existente.

\section{LITERATURA CITADA}

Allendes, J.L., \& Montalva J. 2011. First record of the mite Kuzinia laevis (Dujardin, 1849)

(Acarina: Acaridae). Boletín de Biodiversidad de Chile, 5, 36-38.

Bosch, M., Simon J., Blanché C., \& Molero J. 1997. Pollination ecology in tribe Delphineae ( $\mathrm{Ra}-$ nunculaceae) in W. Mediterranean areas: floral visitors and pollinator behaviour. Lagascalia, 19(1-2), 545-562.

Briscoe, A.D. \& Chittka L. 2001. The Ecology of color vision in insects. Annual Review of Entomology, 46, 471-510.

Buchmann, S.L. 1983. Buzz pollination in angiosperms. In Jones, C.E. \& R.J. Little (Eds.), Handbook of experimental pollination biology, pp. 73- 113. 
Van Nostrand-Rheinhold, New York.

Castro, K. 2012. La difícil situación del abejorro Bombus terrestris en la región de Magallanes 155 más austral del mundo. Geográfica Chilena. http:// www.google.cl/\#output=search\&sclient=psyab\&q=Kur $\mathrm{t}+$ Castro+2012+La+dif\%C3\%ADc il+situac i $\%$ B3n+del+abejorro+m\%C3\%A1s+a ustral +del+mundo\&oq=Kurt+C 10/03/2013.

Dafni, A. \& Shmida A. 1996. The possible ecological implications of the invasion of Bombus terrestris (L.) (Apidae) at Mt. Carmel, Israel. In Matheson, A., S.L. Buchmann, C.O'Toole, P. Westrich \& J.H. Williams (Eds.), The conservation of bees, pp.183-200. Academic Press, London.

Dafni, A. 1998. The threat of Bombus terrestris spread. Bee world, 79, 113-114.

Estay, P. 2007. Bombus en Chile: especies, biología y manejo. Colección Libros INIA № 22.

Goka, K. 1998. Influences of invasive species on native species - will the European bumblebee, Bombus terrestris, being genetic pollution into japanese native species? Bulletin of Biography Society in Japan, 53, 91-101.

Goka, K., Okanave K., Yoneda M., \& Niwa S. 2001. Bumblebee commercialization will cause worldwide migration of parasitic mites. Molecular Ecology, 10, 2095-2099.

Goulson, D. 2003. Effects of introduced bees on native ecosystems. Annual Review of Ecology and Systematics, 34, 1-26.

Goulson, D., Lye G.C., \& Darvill B., 2008. Decline and Conservation of Bumble Bees. Annual Review of Entomology, 53, 362-378.

Groombridge, B. (Ed.). 1992. Global Biodiversity. Status of the Earth's Living Resources. Chapman \& Hall.

Hingston, A. \& McQuillan P., 1999. Displacement of Tasmanian native megachilid bees by the recently introduced bumblebee Bombus terrestris (Linnaeus, 1758) (Hymenoptera: Apidae). Australian Journal of Zoology, 47(1), 59-65.

Inoue, M.N., Yokoyama J., \& Washitani I., 2008. Displacement of Japanese native bumblebees by the recently introduced Bombus terrestris (L.) (Hymenoptera: Apidae). Journal of Insect Conservation, 12, 135-146.

Matsumura, C., Yokoyama J., \& Washitani I., 2004. Invasion status and potential impacts of an invasive alien bumblebee, Bombus terrestris (L.)
(Hymenoptera: Apidae) naturalized in southern Hokkaido, Japan. Global Environmental Research, 8, 55-66.

Montalva, J. 2001. Las posibles implicancias de la introducción de Bombus terrestris (L.) en Chile. http://entomologianet/montalval.htm. 24/02/2013.

Montalva, J., Ruz L., \& Arroyo M.T.K., 2008. Bombus terrestris Linnaeus (Hymenoptera: Apidae). Causas y consecuencias de su introducción. Revista del Jardín Botánico Chagual, 6(6), 13-20.

Montalva, J. \& Ruz L., 2010. Actualización de la lista sistemática de las abejas chilenas (Hymenoptera: Apoidea). Revista Chilena de Entomología, 35, 15-52.

Montalva, J., Dudley L., Arroyo M.K., Retamales H., \& Abramovich H.A., 2011. Geographic distribution and associated flora of native and introduced bumble bees (Bombus spp.). Journal of Apicultural Research, 50(1), 11-21.

Montalva, J. 2012. Bombus terrestris (Linnaeus, 1758). Abejas de Chile. Windows Internet Explorer. http://abejasdechile.blogspot. com/2012/04/ bombus-terrestris.html 24/02/2013.

Morales, C.L. 2007. Introducción de abejorros (Bombus) no nativos: causas, consecuencias ecológicas y perspectivas. Asociación Argentina de Ecología. Ecología Austral, 17, 51-65.

NAPPC (North American Pollinator Protection Campaign). 2006. Importation of Non-Native Bumble Bees into North America: Potential Consequences of Using Bombus terrestris and Other Non-Native Bumble Bees for Greenhouse Crop Pollination in Canada, Mexico, and the United States. A White Paper of the North American Pollinator Protection Campaign. 35 pp. http:// www.pollinator.org./ Resources//BEEIMPORTATION_aug2006. pdf 15/01/2013

O'Toole, C. 1994. Who cares for solitary bees? In Matheson, A. (Ed.), Forage for Bees in an Agricultural Landscape. Cardiff: IBRA, pp. 47-56.

Pérez, V. \& Petersen J., 1989. Notas sobre abejas de la Región Magallánica, Chile (Hymenoptera: Apoidea). Acta Entomológica Chilena, 15, 257-260.

Plischuck, S. \& Lange C.E., 2009. Invassive Bombus terrestris (Hymenoptera: Apidae) parasitized by a flagellate (Euglenozoa: Kinetoplastea) and an neogregarine (Apicomplexa: Neogregari- 
norida). Journal of Invertebrate Pathology, 102(3), 263-265.

Project Noah 2013. Moscardón Bombus terrestris. http://www.projectnoah.org/spottings/ 9404248 20/01/2013

Ruz, L. \& R. Herrera. 2001. Preliminary observations in foraging activities of Bombus dahlbomii and Bombus terrestris (Hym.: Apidae) on native and nonnative vegetation in Chile. Acta Horticulturae, 561, 165-169.

Ruz, L. 2002. Bee pollinators introduced to Chile: a review. In Kevan, P.G. \& V.L. ImperatrizFonseca (Eds.). Pollinating Bees, 2002: The Conservation Link Between Agriculture and Nature. Proceedings Workshop Conservation and Sustainable Use of Pollinators in Agricultute, with Emphasis on Bees. Brasilia Ministry of Environment. Sao Paulo, Brazil. Schmidt-Hempel, P. 1998. Parasites in social insects. Princeton University Press, New Jersey.

Squeo, F.A., Estadés E., Bahamonde N., L.A.Cavieres, Rojas G., Benoit I., Parack E., Fuentes A., Avilés R., Palma A., Solís R.o., Guerrero S., Montenegro G., \& Torres-Mura J.C., 2010. Revisión de la clasificación de especies en categorías de amenazadas en Chile. Revista Chilena de Historia Natural, 85, 511-529.

Thorp, R. 1996. Resource overlap among native and introduced bees in California. In Matheson, A., S.L.C. Buchmann, P.O'Toole, P. Westrich \& I.H. Williams (Eds.), pp.143-151. Academic Press, London.

Torreta, J.P., Medan D., \& Abramovich A.H., 2006.
First record of the invasive bumblebee Bombus terrestris (L.) (Hymenoptera, Apidae) in Argentina. Transactions of the American Entomological Society, 132, 285-289.

Van der Steen, J. 2000. Diseases and parasites relevant to Bombus terrestris L. indoor rearing. In Sommeijer, M.J. \& A. Ruijter (Eds.), Insects Pollination in Geenhouses, pp.3537.Utretch University \& Ambrosiushoeve. Utretch, Hilvarenbeek.

Velthuis, H.H.W. \& Van Doorn S.A., 2006. A century of advances in bumble bee domestication, and the economic and environmental aspects of its commercialization for pollinization. Apidologie, 37, 421-451.

Williams, P.H., Brown M.J.F., Carolan J.C., An J., Goulson D., Aytekin A.M., Best L.R, Byvaltsev A.M., Cederberg B., Dawson R., Huang J., Ito M., Monfaredd A., Raina R.H., SchmidtHempel P., Sheffield C.S., Sima P. \& Xie Z., 2012. Unveiling cryptic species of the bumble bee subgenus Bombus s.str., worldwide with COI barcodes (Hymenoptera: Apidae). Systematics and Biodiversity, 10(1), 21-56. Willmer, P.G., Bataw A.A.A., \& Highes J.P., 1994. The superiority of bumblebees to honeybees as pollinators: insects visits to raspberry flowers. Ecological Entomology, 9, 2271-284.

Yoon, H.J., Kim S.Y., Lee K.Y., Lee S.B., Park I.G. \& Kim I., 2009. Interspecific Hybridization of the Bumblebees Bombus ignitus and Bombus terrestris. International Journal of IndustrialEntomology, 18(1), 41-58. 